ORIGINAL ARTICLE

\title{
A randomised, placebo controlled trial of low level laser therapy for activated Achilles tendinitis with microdialysis measurement of peritendinous prostaglandin $E_{2}$ concentrations
}

\author{
J M Bjordal, R A B Lopes-Martins, V V Iversen
}

Br J Sports Med 2006;40:76-80. doi: 10.1136/bjsm.2005.020842

Background: Low level laser therapy (LLLT) has gained increasing popularity in the management of tendinopathy and arthritis. Results from in vitro and in vivo studies have suggested that inflammatory modulation is one of several possible biological mechanisms of LLLT action.

Objective: To investigate in situ if LLLT has an anti-inflammatory effect on activated tendinitis of the human Achilles tendon.

Subjects: Seven patients with bilateral Achilles tendinitis (14 tendons) who had aggravated symptoms produced by pain inducing activity immediately before the study.

Method: Infrared (904 nm wavelength) LLT (5.4 J per point, power density $20 \mathrm{~mW} / \mathrm{cm}^{2}$ ) and placebo LLLT (O J) were administered to both Achilles tendons in random blinded order.

See end of article for authors' affiliations

Correspondence to: Dr Bjordal, Physiotherapy Science, University of Bergen, Bergen, Norway; jmbjor@broadpark.no

Accepted 1 July 2005
Results: Ultrasonography Doppler measurements at baseline showed minor inflammation through increased intratendinous blood flow in all 14 tendons and measurable resistive index in eight tendons of 0.91 (95\% confidence interval 0.87 to 0.95 ). Prostaglandin $E_{2}$ concentrations were significantly reduced 75,90 , and 105 minutes after active LLLT compared with concentrations before treatment $(p=0.026)$ and after placebo LLLT ( $p=0.009)$. Pressure pain threshold had increased significantly $(p=0.012)$ after active LLLT compared with placebo LLLT: the mean difference in the change between the groups was $0.40 \mathrm{~kg} / \mathrm{cm}^{2}$ (95\% confidence interval 0.10 to 0.70$)$.

Conclusion: LLLT at a dose of $5.4 \mathrm{~J}$ per point can reduce inflammation and pain in activated Achilles tendinitis. LLLT may therefore have potential in the management of diseases with an inflammatory component.
O steoarthritis, tendinitis, and painful spinal disorders are the most common musculoskeletal disorders in modern society. ${ }^{1-4}$ A whole range of different treatments such as locally applied or orally administered drugs, electrotherapies, joint mobilisation techniques, exercise therapy, cognitive behavioural therapies, and alternative treatments are currently in use for these conditions. ${ }^{5-9}$ Nonsteroidal anti-inflammatory drugs (NSAIDs) and steroid injections have been the most prevalent form of treatment for short term pain relief. ${ }^{10-12}$ Most treatments exhibit some pain relieving effect against osteoarthritis and tendinopathy. ${ }^{13}{ }^{14}$ However, it is unclear whether any electrophysical agents have anti-inflammatory effects. A review on the biological effects of ultrasound therapy suggests that there is no evidence that it has any major anti-inflammatory effect. ${ }^{15}$ For low level laser therapy (LLLT) two important biological responses have been targeted as possible mechanisms for its beneficial clinical effects in clinical trials on tendinopathy and osteoarthritis. ${ }^{16}{ }^{17}$ The first possible biological response is a modulating, dose dependent effect on fibroblast metabolism and collagen deposition. This response has been observed in a broad range of controlled studies on cell cultures and animals. ${ }^{18-22}$ The second possible mechanism is similar to that of NSAIDs and steroids. Controlled laboratory trials have revealed that LLLT can reduce inflammation through reduction of $\mathrm{PGE}_{2}$ concentrations and inhibition of cyclo-oxygenase 2 in cell cultures. ${ }^{23}{ }^{24}$ This effect has also been shown in an animal trial, but to our knowledge no study has yet confirmed such an anti-inflammatory effect in humans. ${ }^{25}$ Microdialysis is a technique used to assess peritendinous inflammation in normal and symptomatic tendons. ${ }^{26}$ Although some researchers have questioned the presence of inflammation in chronic tendinopathies, others have shown that structural tendon damage and ruptures correlate significantly with the degree of inflammation. ${ }^{28-30}$ In addition, loading of tendon cells increased the expression of cyclo-oxygenase 2 and the release of prostaglandin $\mathrm{E}_{2}\left(\mathrm{PGE}_{2}\right)$ in vitro and peritendinous $\mathrm{PGE}_{2}$ concentrations in a human experimental model of healthy Achilles tendons. ${ }^{31}$ In healthy subjects, this activity induced release of $\mathrm{PGE}_{2}$ could be blocked by a cyclo-oxygenase 2 inhibitor (celecoxib). ${ }^{27}$ Therefore microdialysis after activity induced inflammation seems to be a suitable method for assessing the anti-inflammatory effect of LLLT in humans.

\section{MATERIALS AND METHODS \\ Patient sample}

Patients were recruited through primary care doctors and physiotherapists. Patients who sought help for their bilateral Achilles pain were targeted, and given the opportunity to participate before standard treatment was started. Inclusion criteria were: signed written informed consent statement; age 20-60 years; bilateral symptoms of pain and tenderness from the Achilles tendons that could be aggravated by some type of physical activity; willingness to perform the type of physical activity that by experience aggravated Achilles tendon pain; pathological sonographic appearance of tendon thickening

Abbreviations: LLLT, low level laser therapy; NSAID, non-steroidal antiinflammatory drug; $\mathrm{PGE}_{2}$, prostaglandin $\mathrm{E}_{2} ; \mathrm{Rl}$, resistive index 
and structural alterations of tendon matrix. Exclusion criteria were: systemic inflammatory disease; previous operations on Achilles tendons; pregnancy; mental inability to understand the consequences of participation; NSAID treatment during the preceding four weeks; steroidal treatment during the preceding six months; monogenic familial hypercholesterolaemia (may cause Achilles tendon xanthomas).

\section{Examination procedure and outcome measures}

Each patient was examined by interview about symptom characteristics, symptom duration, and specific activities that caused aggravation of Achilles tendon symptoms. A physical examination was then performed. After location of the most painful spot identified by finger palpation, pressure pain threshold was measured as the average of three tests with pressure pain algometry. This test was performed by pressing a circular metallic tip of area $1 \mathrm{~cm}^{2}$ with gradually increasing force to the most painful spot of the tendon. The patient was told to respond by saying "stop" when the feeling of pressure changed to pain. Before the experiment, this test was checked on healthy subjects for test-retest reliability; the intraclass correlation coefficient was 0.79 and standard deviation $0.39 \mathrm{~kg} / \mathrm{cm}^{2}$, which is similar to other reliability studies for pressure pain threshold. ${ }^{32-34}$

Functional ability was tested by measuring the mean distance of three repetitions reached in the single leg hop test. $^{35}$ Ultrasonography (Siemens Sonoline G60) was performed to ensure the exact location of tendon pathology. Tendon thickness was measured using calibrated callipers, and structural alteration of the tendon matrix was described. Tendon vascularisation was examined by power Doppler. If possible, peritendinous or intratendinous arterial blood flow velocity was also measured, as they can be used to detect signs of inflammation through the arterial resistive index (RI), which is a measure of vasodilatation and inflammation. ${ }^{36}$ RI is defined as: (systolic peak velocity minus end diastolic velocity)/systolic peak velocity. Values of less than 1 (normal) indicate inflammation.

Microdialysis was performed by first giving a local anaesthetic injection (xylocaine, $20 \mathrm{mg} / \mathrm{ml}$; AstraZeneca, Oslo, Norway) at the insertion site $30 \mathrm{~mm}$ cranially to the centre of pathology. After four to five minutes, the microdialysis membrane (CMA/60, cut off $20 \mathrm{kDa}$, membrane length $30 \mathrm{~mm}$, outer diameter $0.6 \mathrm{~mm}$; CMA, Stockholm, Sweden) was inserted into the peritendinous tissue parallel to the Achilles tendon. Insertion into the tissue is achieved with the help of a slit cannula introducer which leaves the catheter in place when withdrawn. Before withdrawal of the introducer, the position of the membrane was checked by ultrasonography. The microdialysis membrane and tubes were then coupled to a syringe containing isotonic $0.9 \%$ salt

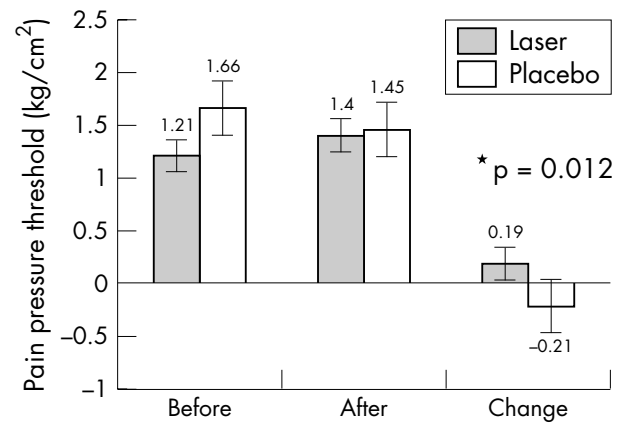

Figure 1 Mean pressure pain thresholds of three recordings from each tendon with their respective $95 \%$ confidence intervals grouped by treatment allocation. water. Syringes for both Achilles tendons were then placed in a syringe pump (CMA/100) and adjusted to give $2 \mu \mathrm{l} / \mathrm{min}$. Samples of the harvested fluid were collected every $15 \mathrm{~min}$ utes. After a stabilisation period of 60 minutes, baseline levels were registered for another 60 minutes before laser treatment was administered. $\mathrm{PGE}_{2}$ concentrations after treatment were recorded for 105 minutes. $\mathrm{PGE}_{2}$ concentration in the dialysate was analysed by a high sensitivity Prostaglandin E2 Enzyme Immunoassay Kit (Electra-box Diagnostica AS, Stockholm, Sweden). The washing, the adding of substrate solutions and stop solutions, and the analyses were performed according to the manufacturers' specifications. For each well, the absorbance was read using a microplate reader (Spectramax; Molecular Devices, Sunnyvale, California, USA) set to $450 \mathrm{~nm}$, with wavelength control.

\section{Intervention}

LLLT was administered by a GaAs laser with an infrared wavelength of $904 \mathrm{~nm}$ (Irradia AB, Stockholm, Sweden) with a laser probe consisting of three laser diodes placed longitudinally $9 \mathrm{~mm}$ apart. On the laser probe, an A/B switch determined whether active or sham irradiation was given. During operation the laser appeared identical for both active and sham irradiation, because of the invisible nature of the infrared laser beam and the same blinking red diode and descending time counter. The output of the laser averaged $10 \mathrm{~mW}$ for each diode, which was tested and calibrated before and after treatment for every patient by technical staff not otherwise involved in the experiment. The peak power for each laser pulse was $10 \mathrm{~W}$ and pulse duration was 200 nanoseconds $\left(10^{-9}\right.$ seconds), delivered by a frequency of $5000 \mathrm{~Hz}$. The spot area was $0.5 \mathrm{~cm}^{2}$ giving a power density (fluence) of $20 \mathrm{~mW} / \mathrm{cm}^{2}$. Each patient was treated for 180 seconds, and the dose of active treatment was $1.8 \mathrm{~J}$ for each of three points along the Achilles tendons, giving a total of $5.4 \mathrm{~J}$ per tendon.

\section{Blinding and randomisation procedure}

The trial was designed to achieve randomised concealed allocation to groups and double blinding. As patients were acting as their own controls, both Achilles tendons were treated, but only one received active treatment. Which side should be treated with the laser probe in switch position $\mathrm{A}$ or $B$ was decided for each patient by drawing one of 10 opaque envelopes containing a written character A or B (five of

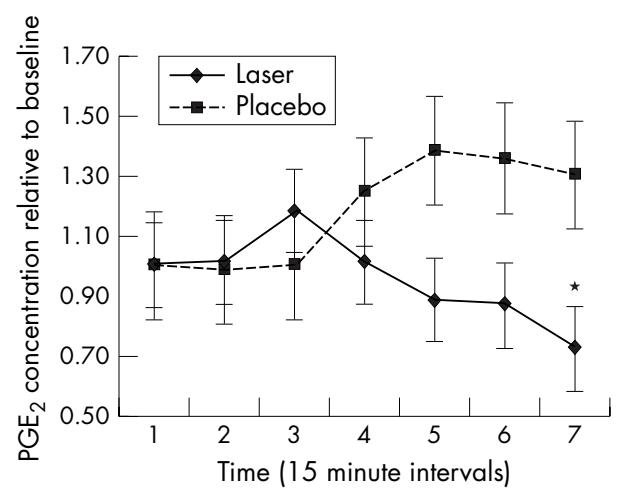

Figure 2 Laser effect on inflammation as measured by prostaglandin $E_{2}$ $\left(\mathrm{PGE}_{2}\right)$ concentrations in peritendinous tissue. Mean post-treatment $\mathrm{PGE}_{2}$ concentrations and their respective $95 \%$ confidence intervals relative to the mean pre-treatment baseline results are shown. Values were recorded at seven consecutive 15 minute intervals (total 165 minutes after treatment), and the significant between-groups difference at the last time point is indicated by an asterisk. 
each). The A/B switch on the laser was then switched to the character drawn, and treatment started for the right leg first. Thus the allocation of tendon to groups was concealed from patients, therapist, and observer. The code of the A/B switch positions on the laser probe was only known to the technical staff who calibrated the laser before and after each patient. The code was not broken until all treatments and analyses were finished.

\section{Ethical approval}

The experiments in this study were performed with the approval of, and in accordance with the regulations laid down by, the regional ethics committee of Western Norway (no 144/03). Participants gave signed informed consent.

\section{RESULTS}

\section{Randomisation procedure}

Four right side and three left side Achilles tendons were given active treatment.

\section{Pressure pain threshold}

There was a small, but non-significant mean decrease $\left(-0.05 \mathrm{~kg} / \mathrm{cm}^{2}\right)$ in the pressure pain threshold from the start of the observation period to immediately before treatment. However, after treatment it had increased by $0.19 \mathrm{~kg} / \mathrm{cm}^{2}$ (95\% confidence interval (CI) 0.04 to 0.34 ) in the laser group, whereas a decrease of $-0.21 \mathrm{~kg} / \mathrm{cm}^{2}(95 \%$ CI +0.04 to -0.46$)$ was recorded for the placebo group from the start to the end the observation period. The mean difference in change was $0.40 \mathrm{~kg} / \mathrm{cm}^{2}$ (95\% CI 0.10 to 0.70 ) (fig 1 ).

\section{Inflammatory marker $\mathrm{PGE}_{\mathbf{2}}$ concentrations}

In the laser group, a small decrease in $\mathrm{PGE}_{2}$ concentration was seen first one hour after treatment, and then gradually decreasing from baseline (1.0) to 0.72 (95\% CI 0.58 to 0.86 ) at the last time point 105 minutes after treatment. For the placebo group, $\mathrm{PGE}_{2}$ concentrations increased gradually in the period after treatment from baseline to 1.30 (95\% CI 1.48 to 1.12 ) at the last observation. For the laser group, there was a significant difference in both the pre-treatment and posttreatment means $(p=0.0269)$ and the difference in change between the groups ( $p=0.0096)$ (fig 2).

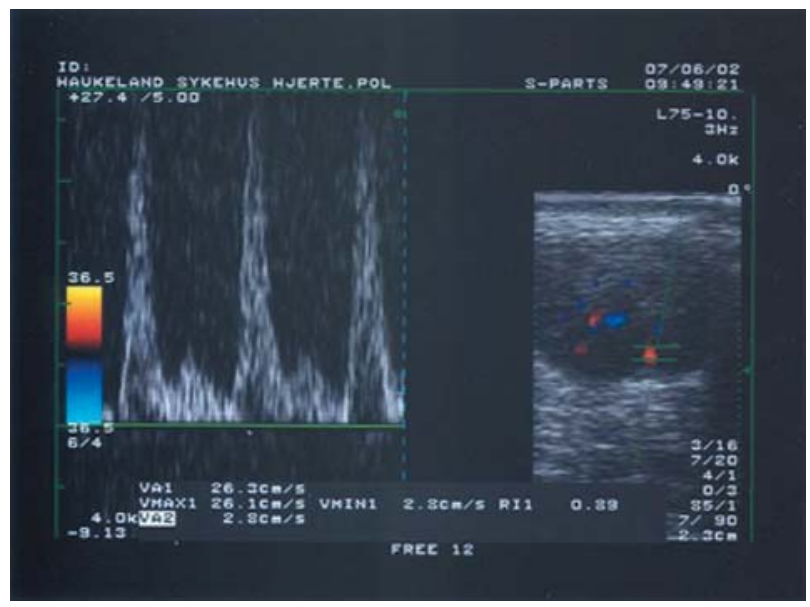

Figure 3 Colour Doppler image of a transverse section of Achilles tendon showing intratendinous blood vessels (right hand frame) and power Doppler (left hand frame) assessment of intratendinous blood flow velocity in systolic peak $(26.1 \mathrm{~cm} / \mathrm{s})$ and diastolic end $(2.8 \mathrm{~cm} / \mathrm{s})$, giving a resistive index of 0.89 , indicating minor inflammation.

\section{Single jump hop test}

Because of the fragile microdialysis membrane and the importance of harvesting fluid meticulously, the single hop test could only be performed before and after microdialysis. Both groups showed a decrease in the single jump hop test after treatment. However, the mean decrease was significantly less $(\mathrm{p}=0.025)$ for the laser treated group $(10.6 \mathrm{~cm}$ (95\% CI 3.5 to 17.7$)$ than for the placebo group $(18.4 \mathrm{~cm}$ (95\% CI 12.3 to 24.5$)$ ).

\section{Ultrasound Doppler scanning}

At baseline all 14 tendons exhibited increased peritendinous and intratendinous blood flow when assessed by colour Doppler in a relaxed position of ankle plantar flexion. At follow up, blood flow measured by colour Doppler seemed to have decreased in all tendons, but no arbitrary assessment was performed. The decreased flow was thought to be a result of the necessary physical inactivity in the experimental set up.

For power Doppler measurements of blood flow, velocity and RI could only be measured at baseline in eight tendons, five in the active LLLT group and three in the placebo LLLT group. The mean RI for these eight tendons indicated a small inflammatory reaction, with RI 0.91 (95\% CI 0.87 to 0.95) (fig 3). No significant differences between groups were observed after treatment for any ultrasound measures.

\section{Side effects and adverse reactions}

The experiment was well tolerated by all patients. Local injection of analgesic (xylocaine) and placement of the microdialysis catheter caused minor transient pain at the time of insertion. Likewise, the application of the pressure pain algometer caused increased tenderness in the area of the Achilles tendon, probably partly because of the repeated pain threshold pressure applications and partly because the effect of the local anaesthetic was tailing off. None of the patients experienced infections or other complications, and they all reported that being bored was a greater problem than the transient pain they experienced.

\section{DISCUSSION}

To our knowledge, other physiotherapy methods have failed to produce relevant anti-inflammatory effects in the laboratory. In this trial, we show that LLLT can suppress inflammation, as measured by a reduction in the inflammatory marker $\mathrm{PGE}_{2}$. In addition, clinical indices of small, but significant improvements in pressure pain and single hop function were observed. The ultrasound measurements were not suitable for detecting differences at follow up mainly because the experimental set up, with hours of physical inactivity, reduces peripheral circulation to a minimum and impairs ultrasound measurements. Still, our baseline ultrasound results may be important for confirming both that pain was actually related to Achilles pathology and a small degree of inflammation was possibly present. ${ }^{37}$ We devised the protocol with prior pain inducing activity, and the ultrasound measurements of RI seem to suggest that a small inflammatory reaction had been induced. Our findings possibly contradict other claims that inflammation is not present in Achilles tendinopathy. ${ }^{28}$ In our opinion, this is not a true contradiction, but rather an elaboration in the sense that inflammation may play a small, but important part in episodes of symptom aggravation. This question remains to be more thoroughly investigated.

The small increase in $\mathrm{PGE}_{2}$ concentration seen in both active and placebo groups immediately after treatment was probably caused by a local reaction to the pressure pain 


\section{What is already known on this topic}

- Low level laser therapy has produced heterogeneous results in clinical trials investigating musculoskeletal pain

- The mechanism underlying the positive results is not known

\section{What this study adds}

- Low level laser therapy can reduce pain by modulating inflammation

threshold testing at this point. Still the results indicate that the anti-inflammatory modulation is present within one hour of application. Our data provide a basis for further exploration and optimisation of LLLT in clinical settings. For tendinopathies, several treatment options exist, but none has been reported to be irrevocably more effective than the others. There are several weaknesses of our study. We only used one fixed LLLT dose and a short follow up of one hour, and therefore the range limits for optimal dose and the duration of the effect remain to be determined. Because of the short duration of the experiment, it would also be inappropriate to conclude that LLLT is effective for Achilles tendinopathy. More laboratory trials are needed to determine optimal dose ranges and intervals between applications, and finally these results must be confirmed in larger clinical trials of longer duration. Because of the diversity of subacute and chronic Achilles tendinopathy, LLLT cannot be recommended as a monotherapy, but should rather be seen as an adjunct to exercises and stretching in an optimal treatment package. ${ }^{38}$

What may be more important is that our findings provide an explanation of the biological mechanism behind the positive clinical LLLT results reported for osteoarthritis and rheumatoid arthritis. ${ }^{39}{ }^{40}$ LLLT is considered by some as one of the most promising non-pharmacological interventions for inflammatory arthritis. ${ }^{41}$ In view of the serious concerns recently raised about side effects of both traditional NSAIDs and cyclo-oxygenase 2 inhibitors in arthritis management, LLLT is a well tolerated alternative that may become a useful treatment for large groups of patients. ${ }^{42}$

\section{CONCLUSION}

In conclusion, these results indicate that LLLT can be used to reduce inflammatory musculoskeletal pain. Because of its rapid action and few side effects, more research is needed to optimise treatment protocols particularly for inflammatory arthritis.

\section{ACKNOWLEDGEMENTS}

This work was supported by a grant from The Norwegian Research Council.

\author{
Authors' affiliations \\ J M Bjordal, V V Iversen, Physiotherapy Science, University of Bergen, \\ Bergen, Norway \\ R A B Lopes-Martins, University of São Paulo, São Paulo, Brazil \\ Competing interests: none declared
}

\section{REFERENCES}

1 Felson DT, Lawrence RC, Dieppe PA, et al. Osteoarthritis: new insights. Part 1: the disease and its risk factors. Ann Intern Med 2000; 133:635-46.

2 Mantyselka $P$, Kumpusalo $E$, Ahonen $R$, et al. Pain as a reason to visit the doctor: a study in Finnish primary health care. Pain 2001;89:175-80.

3 Cherry NM, Meyer JD, Chen Y, et al. The reported incidence of work-related musculoskeletal disease in the UK: MOSS 1997-2000. Occup Med (Lond) 2001;51:450-5.

4 Carmona L, Ballina J, Gabriel R, et al. The burden of musculoskeletal diseases in the general population of Spain: results from a national survey. Ann Rheum Dis 2001:60:1040-5.

5 Jordan KM, Sawyer S, Coakley P, et al. The use of conventional and complementary treatments for knee osteoarthritis in the community. Rheumatology (Oxford) 2004;43:381-4

6 Johansson K, Adolfsson L, Foldevi M. Attitudes toward management of patients with subacromial pain in Swedish primary care. Fam Pract 1999; 16:233-7.

7 Assendelft W, Green S, Buchbinder R, et al. Tennis elbow. BMJ 2003;327:329.

8 Deeks JJ, Smith LA, Bradley MD. Efficacy, tolerability, and upper gastrointestinal safety of celecoxib for treatment of osteoarthritis and rheumatoid arthritis: systematic review of randomised controlled trials. BMJ 2002;325:619.

9 Non-steroidal anti-inflammatory drugs for low back pain. Cochrane Library. Oxford: Update Software, 2001

10 van der Windt DAWM, Koes B, de Jong BA, et al. Shoulder disorders in general practice: incidence, patient characteristics an management. Ann Rheum Dis 1995:54:959-64.

11 Piligian G, Herbert R, Hearns M, et al. Evaluation and management of chronic work-related musculoskeletal disorders of the distal upper extremity. Am J Ind Med 2000;37:75-93.

12 Assendelft W, Green S, Buchbinder R, et al. Tennis elbow (lateral epicondylitis). Clin Evid 2003:1388-98.

13 Jordan KM, Arden NK, Doherty M, et al. EULAR Recommendations 2003: an evidence based approach to the management of knee osteoarthritis: Report of a Task Force of the Standing Committee for International Clinical Studies Including Therapeutic Trials (ESCISIT). Ann Rheum Dis 2003;62:1145-55

14 Smidt N, van der Windt DA, Assendelft WJ, et al. Corticosteroid injections, physiotherapy, or a wait-and-see policy for lateral epicondylitis: a randomised controlled trial. Lancet 2002;359:657-62.

15 Baker KG, Robertson VJ, Duck FA. A review of therapeutic ultrasound: biophysical effects. Phys Ther 2001;81:1351-8.

16 Bjordal JM, Couppe C, Chow RT, et al. A systematic review of low level laser therapy with location-specific doses for pain from chronic joint disorders. Aust J Physiother 2003:49:107-16.

17 Bjordal J, Couppé C, Ljunggreen A. Low level laser therapy for tendinopathy. Evidence of a dose-response pattern. Phys Ther Rev 2001;6):91-9.

18 Loevschall H, Arenholt-Bindslev D. Effect of low level diode laser irradiation of human oral mucosa fibroblasts in vitro. Lasers Surg Med 1994;14:347-54.

19 Pereira AN, Eduardo Cde P, Matson E, et al. Effect of low-power laser irradiation on cell growth and procollagen synthesis of cultured fibroblasts. Lasers Surg Med 2002;31:263-7.

20 Almeida-Lopes L, Rigau J, Amaro Zangaro R, et al. Comparison of the low level laser therapy effects on cultured human gingival fibroblasts proliferation using different irradiance and same fluence. Lasers Surg Med $2001 ; 29: 179-84$.

21 Reddy GK, Stehno-Bittel L, Enwemeka CS. Laser photostimulation of collagen production in healing rabbit achilles tendon. Lasers Surg Med 1998;22:281-7.

22 Yu W, Naim JO, Lanzafame RJ. Effects of photostimulation on wound healing in diabetic mice. Lasers Surg Med 1997;20:56-63.

23 Honmura A, Ishii A, Yanase M, et al. Analgesic effect of GaAlAs diode laser irradiation on hyperalgesia in carrageenin-induced inflammation. Lasers Surg Med 1993:13:463-9.

24 Sakurai Y, Yamaguchi M, Abiko Y. Inhibitory effect of low-level laser irradiation on LPS-stimulated prostaglandin E2 production and cyclooxygenase-2 in human gingival fibroblasts. Eur J Oral Sci 2000; 108:29-34.

25 Medrado AR, Pugliese LS, Reis SR, et al. Influence of low level laser therapy on wound healing and its biological action upon myofibroblasts. Lasers Surg Med 2003;32:239-44

26 Langberg H, Skovgaard D, Karamouzis M, et al. Metabolism and inflammatory mediators in the peritendinous space measured by microdialysis during intermittent isometric exercise in humans. J Physiol 1999;515:919-27.

27 Langberg $H$, Boushel R, Skovgaard D, et al. Cyclo-oxygenase- 2 mediated prostaglandin release regulates blood flow in connective tissue during mechanical loading in humans. J Physiol 2003;551:683-9.

28 Alfredson $\mathrm{H}$, Lorentzon R. Chronic tendon pain: no signs of chemical inflammation but high concentrations of the neurotransmitter glutamate. Implications for treatment? Curr Drug Targets 2002;3:43-54.

29 Ljung BO, Forsgren S, Friden J. Substance $\mathrm{P}$ and calcitonin gene-related peptide expression at the extensor carpi radialis brevis muscle origin: implications for the etiology of tennis elbow. J Orthop Res 1999;17:554-9.

30 Li Z, Yang G, Khan M, et al. Inflammatory response of human tendon fibroblasts to cyclic mechanical stretching. Am J Sports Med 2004;32:435-40.

31 Wang JH, Jia F, Yang G, et al. Cyclic mechanical stretching of human tendon fibroblasts increases the production of prostaglandin E2 and levels of 
cyclooxygenase expression: a novel in vitro model study. Connect Tissue Res 2003;44:128-33.

32 Smidt N, van der Windt DA, Assendelft WJ, et al. Interobserver reproducibility of the assessment of severity of complaints, grip strength, and pressure pain threshold in patients with lateral epicondylitis. Arch Phys Med Rehabil 2002;83:1145-50.

33 Maquet D, Croisier JL, Demoulin C, et al. Pressure pain thresholds of tender point sites in patients with fibromyalgia and in healthy controls. Eur J Pain 2004;8:111-17.

34 Antonaci F, Sand T, Lucas GA. Pressure algometry in healthy subjects: interexaminer variability. Scand J Rehabil Med 1998;30:3-8.

35 Ageberg E, Zatterstrom R, Moritz U. Stabilometry and one-leg hop test have high test-retest reliability. Scand J Med Sci Sports 1998;8:198-202.

36 Terslev L, Torp-Pedersen S, Qvistgaard E, et al. Estimation of inflammation by Doppler ultrasound: quantitative changes after intra-articular treatment in rheumatoid arthritis. Ann Rheum Dis 2003;62:1049-53.

37 Ohberg L, Lorentzon R, Alfredson $\mathrm{H}$. Eccentric training in patients with chronic Achilles tendinosis: normalised tendon structure and decreased thickness at follow up. Br J Sports Med 2004;38:8-11; discussion 11.

38 Fahlstrom $M$, Jonsson $P$, Lorentzon $R$, et al. Chronic Achilles tendon pain treated with eccentric calf-muscle training. Knee Surg Sports Traumatol Arthrosc 2003; 11:327-33.

39 Gur A, Cosut A, Sarac AJ, et al. Efficacy of different therapy regimes of lowpower laser in painful osteoarthritis of the knee: a double-blind and randomized-controlled trial. Lasers Surg Med 2003;33:330-8.

40 Ottawa Panel Evidence-Based Clinical Practice Guidelines for Electrotherapy and Thermotherapy Interventions in the Management of Rheumatoid Arthritis in Adults. Phys Ther 2004;84:1016-43.

41 Li LC. What else can I do but take drugs? The future of research in nonpharmacological treatment in early inflammatory arthritis. J Rheumatol Suppl 2005;72:21-4.

\section{COMMENTARY}

This study is of importance in that it provides direct evidence, for the first time in human volunteers, that low level laser therapy (LLLT) with $904 \mathrm{~nm}$ laser reduces the production of $\mathrm{PGE}_{2}$ after local, transcutaneous application for Achilles tendinitis. It adds significantly to other studies that have shown a reduction in $\mathrm{PGE}_{2}$ in animal models and cell cultures. Several mechanisms have been proposed to explain how pain is modulated by LLLT. This study provides additional support for an anti-inflammatory effect. Given the serious concerns with long term intake of NSAIDs, the potential for application of LLLT for Achilles tendinitis and inflammatory conditions, such as rheumatoid arthritis, is also raised. Interestingly, this study suggests an inflammatory component to Achilles tendinitis not previously recognised. LLLT for the management of Achilles tendinitis remains to be established in clinical trials. Although the numbers are limited, nevertheless, this study provides a model for further research.

R Chow

Castle Hill Medical Centre, Castle Hill, NSW, Australia; rtchow@bigpond.net.au 